\title{
Histopathologic Evaluations of the Lingual Artery in Healthy Tongue of Adult Cadaver
}

\author{
Mi Jin Mun ${ }^{1}$ Chang-Hoon Lee ${ }^{2} \cdot$ Byung-Joo Lee ${ }^{3}$ Jin-Choon Lee ${ }^{3} \cdot$ Jeon Yeob Jang ${ }^{3}$ Sung Hoon Jung ${ }^{1}$ \\ Soo-Geun Wang ${ }^{3}$ \\ ${ }^{1}$ Department of Otorhinolaryngology-Head and Neck Surgery, Busan St. Mary's Hospital, Busan; \\ ${ }^{2}$ Department of Pathology, Pusan National University Hospital, Pusan National University School of Medicine, Busan; \\ ${ }^{3}$ Departement of Otorhinolaryngology-Head and Neck Surgery, Pusan National University School of Medicine, Busan, Korea
}

Objectives. To clarify the anatomical distribution of the lingual artery in normal adult subjects through histopathologic evaluations.

Methods. Eighteen healthy cadaveric tongues were used to produce 8 paraffin-embedded tissue sections each. Length from midline raphe, depth from dorsum of tongue and the whole transverse length tongue were measured.The lateral distance, depth, and proportion of lateral distance of deep lingual artery were determined from tip to base of tongue gradually. Lateral distance is length from median raphe to the center of deep lingual artery lumen. Depth is vertical distance from dorsal surface of tongue to the center of deep lingual artery. Proportion of lateral distance is obtained by dividing lateral distance with transverse length from median raphe to lateral border of tongue. The degree of symmetry between right and left sides and the difference between selected spots were evaluated.

Results. Right and left sides of the lingual artery were symmetric. The lingual artery was lateralized as it run posterior. The lingual artery runs gradually deeper from the surface as it goes near the base of tongue. Both length and depth of the lingual artery gradually increased between $0 \%-75 \%$ of the mobile tongue, but $75 \%-100 \%$ zone of the lingual artery showed no significant difference. There was no anastomosis between right and left side of the lingual arteries. The lingual artery was located within $50 \%$ of the transverse length of tongue from median raphe.

Conclusion. The present study reveals 3-dimensional information on the anatomical distributions of the lingual artery in normal adult subjects. These findings gives us beneficial information about the handling of the lingual artery during oral and base of tongue-related surgery.

Keywords. Blood Supply, Tongue; Surgery, Oral; Anatomy and Histology, Tongue

\section{INTRODUCTION}

The lingual artery is distributed in base of tongue and mobile tongue and susceptible damage during operation. Such injury can lead to immediate or delayed hemorrhage, which can be life

\footnotetext{
- Received July 13, 2015

Revised August 20, 2015

Accepted September 16, 2015

- Corresponding author: Soo-Geun Wang

Department of Otorhinolaryngology-Head and Neck Surgery, Pusan

National University Hospital, Pusan National University School of

Medicine, 179 Gudeok-ro, Seo-gu, Busan 49241, Korea

Tel: +82-51-240-7335, Fax: +82-51-246-8668

E-mail: wangsg@pusan.ac.kr
}

threatening. If both lingual arteries are damaged, necrosis of mobile tongue below damaged point is inevitable. Knowledge of anatomical distribution of the lingual artery is crucial during surgery such as partial glossectomy or tongue base surgery to reduce complications. It is also helpful to decide resection margin and reconstruction.

The lingual artery originates from the external carotid artery just above the superior thyroid artery and runs into the intrinsic muscle of tongue through the posterior border of the hyoglossus muscle. The lingual artery gives first branch, dorsal lingual artery, while passing the hyoglossus muscle. It proceeds anterosuperiorly and gives sublingual artery at the anterior border of hyoglossus muscle. After giving sublingual artery, the lingual ar-

Copyright $@ 2016$ by Korean Society of Otorhinolaryngology-Head and Neck Surgery

This is an open-access article distributed under the terms of the Creative Commons Attribution Non-Commercial License (http://creativecommons.org/licenses/by-nc/4.0)

which permits unrestricted non-commercial use, distribution, and reproduction in any medium, provided the original work is properly cited. 
tery shifts to deep lingual artery. Deep lingual artery runs through inferior portion of mobile tongue giving multiple perforating branches toward dorsum of the tongue. The lingual artery can be subdivided into 4 segments. The first segment is original segment which starts from hyoid bone to the point where dorsal lingual artery is given. The 2 nd part, hyoglossus segment and the 3rd part, ascending segment is divided where the sublingual artery originates. Deep lingual artery is designated as horizontal segment [1].

There were several previous studies to analyze the course of the lingual artery and the anatomical markers. Hou et al. [2,3] used computed tomographic angiography to analyze anatomic relationship between the lingual artery and lingual markers and to set safety space for functional surgery on the tongue. Kimura et al. [4] used Doppler sonography to evaluate the lingual artery at the midpoint of tongue. Lopez et al. [5] studied about vascular territory of the lingual artery using selective ink injections in 15 fresh cadaver heads. But none of these studies researched histologic features of the lingual artery on mobile tongue. In this study, we made 18 healthy cadaveric tongues into 8 coronal sections each to clarify the anatomical distribution of the lingual artery in normal Korean subjects by light microscopic observation.

\section{MATERIALS AND METHODS}

\section{Subjects}

For this study, eighteen healthy cadaveric tongues were used to get 8 pieces of paraffin-embeded tissue section each. Fifteen cases were male, 3 cases were female. Median age was 62 years old ranging from 31 to 88 years. Before producing histologic section, we chose one specimen and performed intramuscular dissection of the tongue to identify the course of the lingual artery. The neck has been partially dissected by medical students and further dissections were performed by the authors. After identifying external carotid artery, we could find the lingual artery just above the superior thyroid artery at the level of hyoid bone. We removed hyoglossus muscle to expose the lingual artery and careful intramuscular dissection was done along the artery. The lingual artery enters base of tongue at greater cornu of hyoid bone and runs along the medial surface of hyoglossus

\section{H I G G H L I}

- Anatomy of the lingual artery was examined in 18 healthy human cadaveric tongues.

- The artery in right and left side was symmetric.

- The artery was positioned laterally and then go deeply posterior to the base of tongue.

- The artery had no communication between right and left sides and was located within $50 \%$ from median raphe.

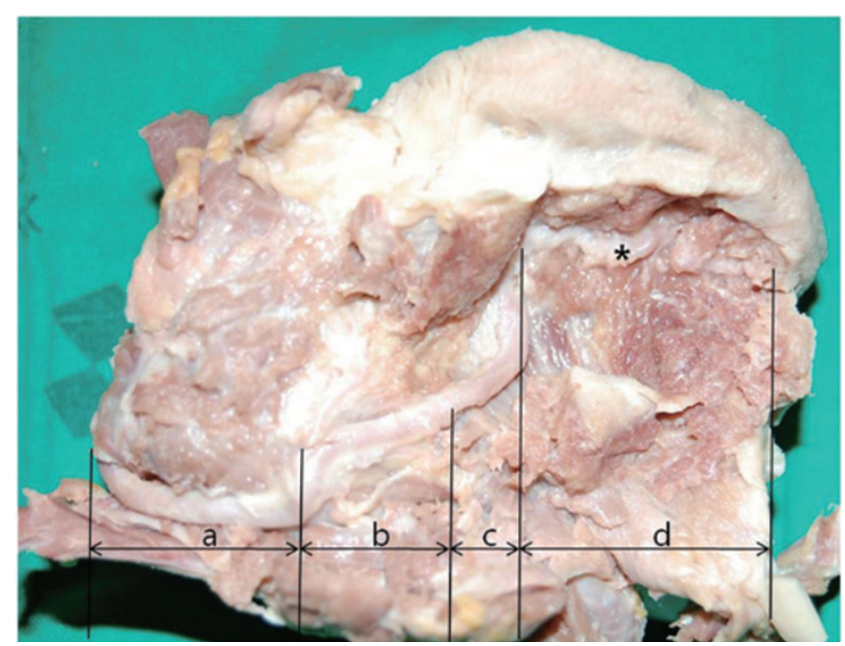

Fig. 1. Cadaveric dissection of the lingual artery. Intramuscular dissection is done to identify anatomical distribution of the lingual artery. (a) Original segment which is placed around greater horn of hyoid bone. (b) Segment of hyoglossus. The lingual artery runs through hyoglossus muscle. (c) Ascending segment. The lingual artery ascends through intrinsic muscles of mobile tongue. (d) Horizontal segment, also called as a deep lingual artery (marked as an asterisk) which runs inferior surface of mobile tongue.

muscle. It proceeds antero-superiorly and shifts as a deep lingual artery at mobile portion of the tongue which runs along the inferior surface of the mobile tongue (Fig. 1). This specimen was not included in histologic slides. Eighteen cadaveric tongues were measured the lengths from the tip of the tongue to foramen cecum. Each tongue was divided into 8 coronal sections according to the dorsal lengths of each tongue. We designated tongue tip as a ' $0 \%$ ' spot and foramen cecum as a ' $100 \%$ ' spot and acquired tissue sections at $12.5 \%, 25.0 \%, 37.5 \%, 50.0 \%$, $62.5 \%, 75.0 \%, 87.5 \%$, and $100 \%$ spots. Tissue cutting was performed along the vertical plane of dorsal surface of the tongue (Fig. 2A).

The Formalin fixed, paraffin-embedded giant tissue slides were produced and hematoxylin-eosin stains were added. The slides were examined under light microscope. The length from midline raphe, depth (D) from dorsal surface of tongue and the whole transverse length of tongue were measured using Storz E-2410 caliper (Storz, St. Louis, MO, USA) but perforating branches of the lingual artery were excluded. The lateral distance (LD), D, and proportion of LD (P) of deep lingual artery were determined from tip to base of tongue gradually. $\mathrm{LD}$ is length from median raphe to the center of deep lingual artery lumen. $\mathrm{D}$ is vertical distance from dorsal surface of tongue to the center of deep lingual artery. $\mathrm{P}$ is obtained by dividing $\mathrm{LD}$ with transverse length from median raphe to lateral border of tongue (Fig. 2B). Thereafter, the degree of symmetry between right and left side and the difference between selected zones ( $25 \%, 50 \%, 75 \%$, and $100 \%$ spots from tip to foramen cecum) were evaluated. We reconstructed 3-dimensional images of lin- 

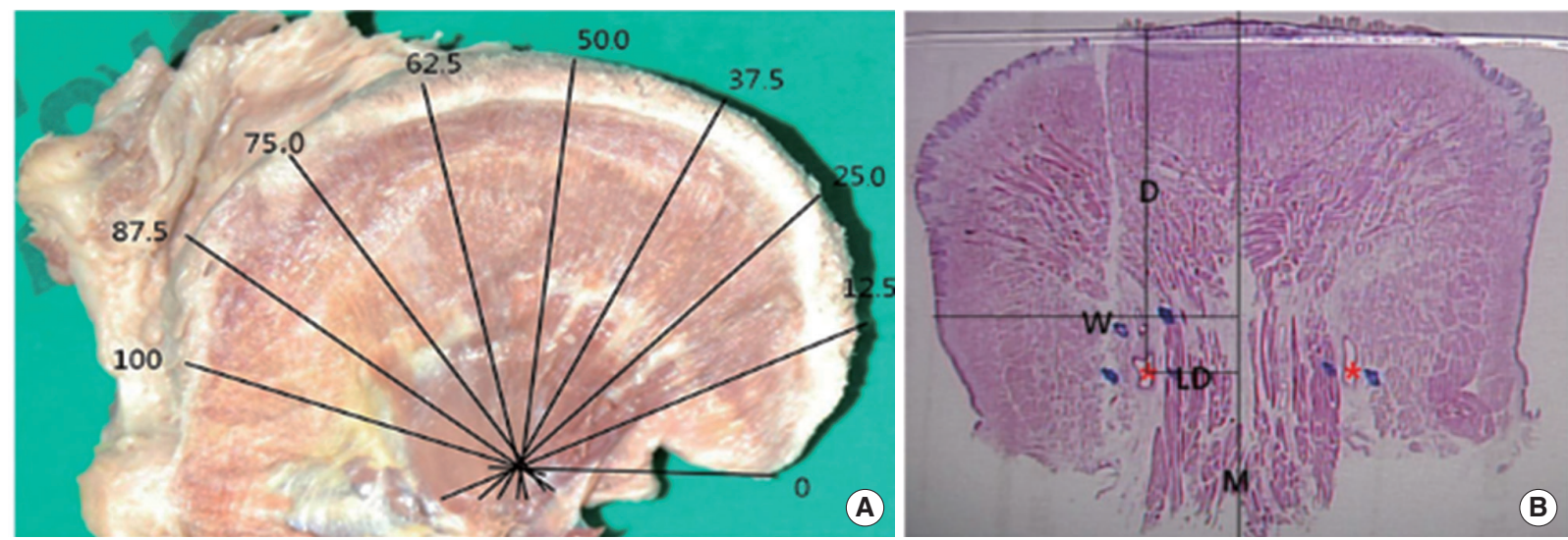

Fig. 2. (A) Method of tissue cutting. The mobile tongue is cut into 8 pieces. (B) Cut surface of mobile tongue (62.5\% portion from tip of tongue). 'LD' means the lateral distance of the lingual artery from median raphe. ' $\mathrm{D}$ ' means the depth of the lingual artery from dorsum of tongue. 'W' means half of total horizontal length of tongue. ' $M$ ' means median raphe. The lingual artery is marked with asterisk (H\&E, $\times 40)$.

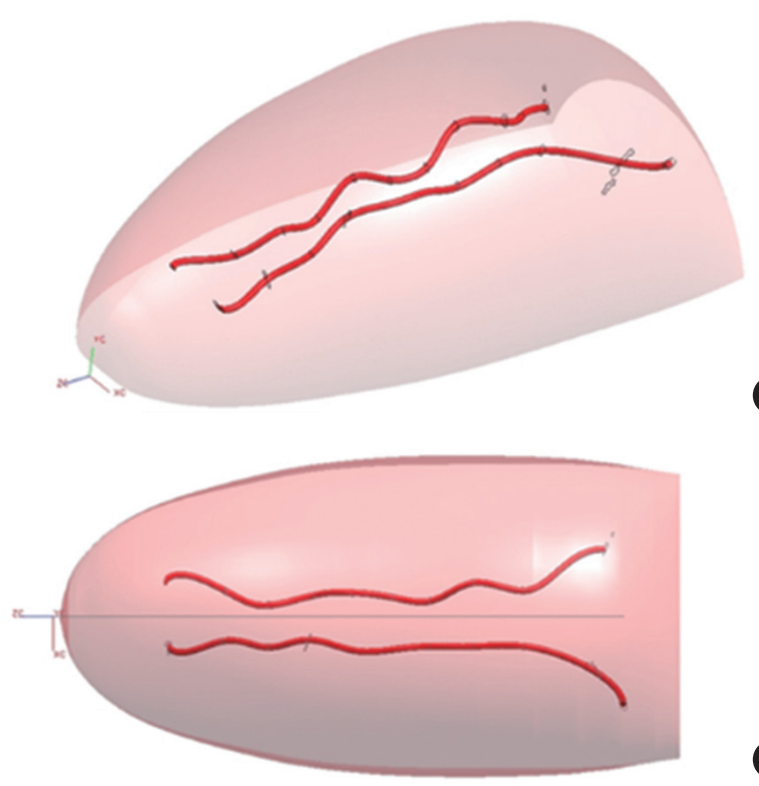

Fig. 3. Three-dimensional reconstruction of the lingual artery by Unigraphics ver. 2.0 (Unigraphics Solutions Inc., Maryland Heights, MO, USA). Bilateral lingual arteries are more lateralized (B) and placed deeper $(A)$ as it runs from tip to base of tongue.

gual artery using Unigraphics ver. 2.0 (Unigraphics Solutions Inc., Maryland Heights, MO, USA) based on data from the representative case (Fig. 3).

\section{Statistical analysis}

The differences between the right and left side locations of the lingual artery were investigated by Wilcoxon sign ranks test. The differences in $25 \%, 50 \%, 75 \%$, and $100 \%$ spots mean values of LD, D, and P were analyzed using Kruskal-Wallis test. Oneto-one comparison of mean values of selected spots (which were $25 \%, 50 \%, 75 \%$, and $100 \%$ ) were performed using Wilcoxon sign ranks test. All differences were defined as significant
Table 1. Lateral distance of the lingual artery on each location

\begin{tabular}{lrrr}
\hline \multirow{2}{*}{ Portion (\%) } & \multicolumn{2}{c}{ Lateral distance $(\mathrm{mm})$} & \multirow{2}{*}{ P-value } \\
\cline { 2 - 3 } & Right side & Left side & \\
\hline 12.5 & $4.6 \pm 2.5$ & $4.9 \pm 2.7$ & 0.488 \\
25.0 & $5.1 \pm 0.9$ & $5.1 \pm 2.9$ & 0.094 \\
37.5 & $6.9 \pm 2.3$ & $6.9 \pm 1.8$ & 0.263 \\
50.0 & $6.6 \pm 2.3$ & $6.7 \pm 2.1$ & 0.549 \\
62.5 & $8.3 \pm 2.4$ & $7.1 \pm 2.4$ & 0.677 \\
75.0 & $8.8 \pm 4.0$ & $9.8 \pm 2.8$ & 0.787 \\
87.5 & $10.5 \pm 4.0$ & $10.8 \pm 4.5$ & 0.253 \\
100 & $11.0 \pm 4.8$ & $12.2 \pm 5.9$ & 0.503 \\
\hline
\end{tabular}

Values are presented as mean \pm SD.

for a $P$-value less than 0.05 . These analyses were performed using the statistical software PASW SPSS ver. 18.0 (SPSS Inc., Chicago, IL, USA).

\section{RESULTS}

\section{Lateral distance of the lingual artery}

The average LD from midline raphe of the tongue were listed on Table 1. LD of $12.5 \%$ spot was the shorter than any other values (right side, $4.6 \pm 2.5 \mathrm{~mm}$; left side, $4.9 \pm 2.7 \mathrm{~mm}$ ) while $\mathrm{LD}$ of foramen cecum was the largest one (right side, $11.0 \pm 4.8$ $\mathrm{mm}$; left side, $12.2 \pm 5.9 \mathrm{~mm}$ ). There was no significant difference between right and left side $(P>0.05)$. LD was gradually increased as lingual artery runs toward foramen cecum (Fig. 4A, B). Statistical analysis of LD at $25 \%, 50 \%, 75 \%$, and $100 \%$ spots using Kruskal-Wallis test showed significant difference in right and left side $(P<0.05)$. On one-to-one comparison using Wilcoxon sign rank test, $25 \%$ spot was significantly different from $50 \%$ and $100 \%$ spots and so was $50 \%$ spot from $100 \%$ in right side $(P<0.05)$. There was no significant difference between $25 \%-75 \%, 50 \%-75 \%$, and $75 \%-100 \%$ spots $(P>0.05)$. In the 

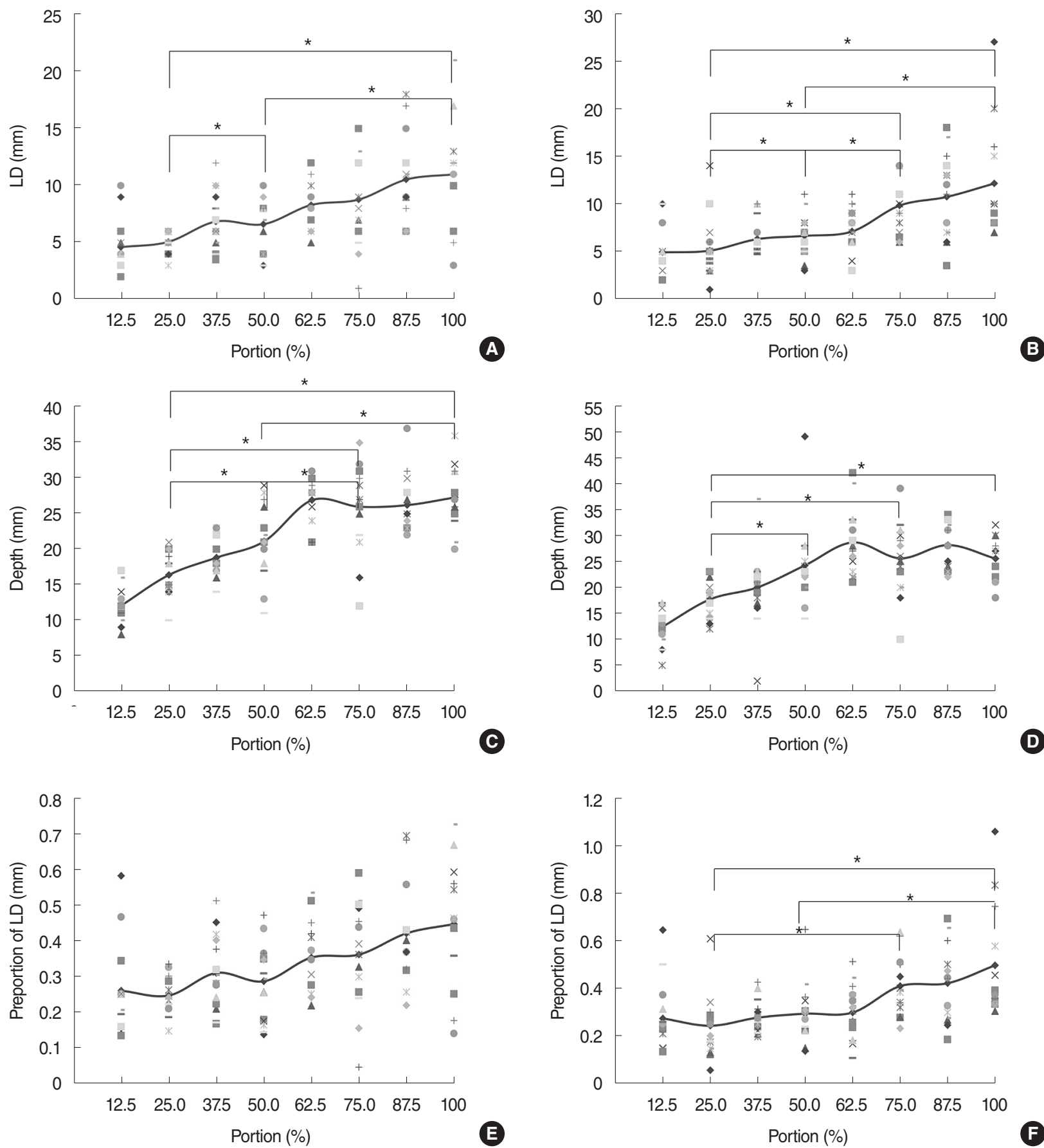

Fig. 4. (A, B) The LD of the lingual artery from median raphe. The graph showed gradual increase as it runs toward foramen cecum. The linear curve is obtained from average of LD. (C, D) The depth of the lingual artery. The lingual artery placed deeper in foramen cecum than tongue tip. The graph gradually increased as it approaches to tongue base. The linear curve is obtained from average of depth. (E, F) The proportion of LD. This parameter shows gradual increase but statistically no significant differences are present on right side. The left side, on the other hand, shows definite difference which is marked as an asterisk. The linear curve is obtained by average of proportion of LD. (A, C, E) Right side. (B, D, F) Left side. LD, lateral distance. ${ }^{*} P<0.05$.

left side however, there were significant differences in $25 \%-$ $50 \%, 25 \%-75 \%, 25 \%-100 \%, 50 \%-75 \%$, and $50 \%-100 \%$ comparison $(P<0.05)$. Only the $75 \%-100 \%$ spot showed no difference in the left side $(P>0.05)$.

\section{Depth of the lingual artery}

Mean values of $\mathrm{D}$ were shown in Table 2. There was no difference between the right and left sides $(P>0.05)$. Mean values of $\mathrm{D}$ at $12.5 \%$ spot were $12.1 \pm 2.5 \mathrm{~mm}$ (right) and $12.5 \pm 3.8 \mathrm{~mm}$ 
Table 2. Depth of the lingual artery on each location

\begin{tabular}{llll}
\hline \multirow{2}{*}{ Portion (\%) } & \multicolumn{2}{c}{ Depth $(\mathrm{mm})$} & \multirow{2}{*}{$P$-value } \\
\cline { 2 - 3 } & Right side & Left side & \\
\hline 12.5 & $12.1 \pm 2.5$ & $12.4 \pm 3.8$ & 0.167 \\
25.0 & $16.4 \pm 3.2$ & $17.7 \pm 3.5$ & 0.556 \\
37.5 & $18.8 \pm 2.5$ & $20.0 \pm 6.9$ & 0.247 \\
50.0 & $21.1 \pm 45$ & $24.3 \pm 7.7$ & 0.392 \\
62.5 & $26.9 \pm 3.4$ & $28.7 \pm 6.0$ & 0.539 \\
75.0 & $26.0 \pm 6.4$ & $25.6 \pm 6.9$ & 0.412 \\
87.5 & $26.2 \pm 4.3$ & $28.1 \pm 4.4$ & 0.441 \\
100 & $27.3 \pm 4.5$ & $25.5 \pm 4.2$ & 0.234 \\
\hline
\end{tabular}

Values are presented as mean \pm SD.

(left) and mean values of $\mathrm{D}$ at foramen cecum were $27.3 \pm 4.5$ $\mathrm{mm}$ (right) and $25.5 \pm 4.2 \mathrm{~mm}$ (left). The lingual artery placed deeper as it run from tongue tip to the foramen cecum (Fig. 4C, D). There was significant difference in comparison between $25 \%, 50 \%, 75 \%$, and $100 \%$ spots using Kruskal wallis test $(P<0.05)$. On Wilcoxon sign rank test, the mean values of $\mathrm{D}$ were significantly different except $75 \%-100 \%$ comparison in the right side $(P<0.05)$. No significant differences were observed between $50 \%-75 \%, 50 \%-100 \%$, and $75 \%-100 \%$, but D of $25.0 \%$ was significantly different with $50 \%, 75 \%$, and $100 \%$.

\section{Proportion of lateral distance of the lingual artery}

The mean values of $\mathrm{P}$ were shown in Table 3 . $\mathrm{P}$ of $12.5 \%$ was closer to midline than other spot (right, $26 \% \pm 13 \%$; left, $27 \%$ $\pm 15 \%$ ), $\mathrm{P}$ of foramen cecum were $45 \% \pm 18 \%$ in the right side and $50 \% \pm 24 \%$ in the left side which located farthest from the midline (Fig. 4E, F). No significant differences were observed between sides $(P>0.05)$. The average $\mathrm{P}$ of $25 \%, 50 \%, 75 \%$, and $100 \%$ spots showed significant difference in Kruskal-wallis test $(P<0.05)$. On Wilcoxon sign rank test, there were no significant difference in the right side, but in the left side, $25 \%-75 \%$, $25 \%-100 \%$, and $50 \%-100 \%$ comparisons showed significant difference $(P<0.05)$.

We reconstructed 3-dimensional images of lingual artery using Unigraphics ver. 2.0 based on data from the representative case (Fig. 3). Both sides of the lingual artery were lateralized from the median raphe and placed deeper from the surface until they reach foramen cecum. Right and left sides of the lingual artery were symmetric.

\section{DISCUSSION}

Oral cavity is limited space so only a small amount of bleeding can disturb surgical fields. Tongue has excellent mobility and abundant vasculatures. Massive bleeding can occur when main trunk of the lingual artery is damaged and ligation is difficult due to limited surgical field [6,7].Thus, it is important to prevent
Table 3. Proportion of lateral distance of the lingual artery on each level

\begin{tabular}{lccc}
\hline \multirow{2}{*}{ Portion (\%) } & \multicolumn{2}{c}{ Proportion of lateral distance (\%) $^{\text {a) }}$} & \multirow{2}{*}{ P-value } \\
\cline { 2 - 3 } & Right side & Left side & \\
\hline 12.5 & $26 \pm 13$ & $27 \pm 15$ & 0.499 \\
25.0 & $25 \pm 5$ & $24 \pm 13$ & 0.114 \\
37.5 & $31 \pm 9$ & $28 \pm 7$ & 0.182 \\
50.0 & $29 \pm 1$ & $29 \pm 12$ & 0.463 \\
62.5 & $35 \pm 1$ & $30 \pm 11$ & 0.799 \\
75.0 & $36 \pm 15$ & $41 \pm 12$ & 0.79 \\
87.5 & $42 \pm 15$ & $42 \pm 16$ & 0.209 \\
100 & $45 \pm 18$ & $50 \pm 24$ & 0.594 \\
\hline
\end{tabular}

Values are presented as mean \pm SD.

a)Proportion of lateral distance=lateral distance of lingual artery from median raphe/0.5 of total horizontal length of tongue.

excessive bleeding by preserving the lingual artery. In this study, we used eighteen cadaveric tongues to clarify anatomical distribution of the lingual artery by light microscopic observation.

There were a few studies about anatomical distributions of the lingual artery using computed tomographic angiography, Doppler sonography and cadaveric tongue [2-5,8]. Hou et al. [2] analyzed the lingual artery to define safety space for functional surgery of the tongue using computed tomographic angiography. He proposed V-zone of the tongue which is similar with our findings [2], but most of the reports focused on base of tongue and lack an explicit description of the lingual artery morphology and topography at the level of the tongue.

We tried to define useful surgical markers to facilitating a surgeon's ability to avoid intraoperative lingual artery injury. We used median raphe, foramen cecum, tongue tip, and dorsal surface of the tongue as anatomical markers because they were relatively constant at any position. We measured the length of the dorsal surface of the tongue from tongue tip to foramen cecum then divided it into 8 coronal sections and produced histopathologic slides. D of the lingual artery from the dorsal surface of the tongue is gradually increased until the lingual artery reaches $75 \%$ of length of tongue from tongue tip. From there, D of the lingual artery remains stable to foramen cecum. $\mathrm{D}$ of the lingual artery varies with the position of the tongue, so measured value itself cannot be used in surgical fields which require traction into multidirections. Operator should keep in mind that the lingual artery located deeper pathway vertically from the surface as it reaches tongue base. The lingual artery runs lateral direction until three-fourths of tongue length. LD of the lingual artery from median raphe varies with the position of the tongue, too. $\mathrm{P}$ remains constant during forward traction of the tongue so it is helpful in surgical fields. The lingual artery is located within $50 \%$ of transverse length of tongue from median raphe. Surgical resection that does not exceed the ranges mentioned above is relatively safe. If the tumor extends more than a half of transverse length from lateral border in one side, the risk of the lin- 
gual artery damage increases. The area between lingual arteries is relatively safe. The mean $\mathrm{LD}$ at foramen cecum are $11.0 \pm 4.8$ $\mathrm{mm}$ in the right side and $12.2 \pm 5.9 \mathrm{~mm}$ in the left side. This is consistent with Hou et al. [2]'s findings that surgery performed within approximately $15 \mathrm{~mm}$ of the tongue midline is relatively safe. Both sides of the lingual artery run symmetric and there is no transseptal anastomosis between sides like Houseman et al. [9]'s findings. However, the limitation of this study is that tissue shrinkage can occur during formalin fixation and microscopic slide production. The degree of shrinkage can differ according to contractility of the tissue. Tongue tissue can shrink due to intrinsic muscle's contraction [10].

In conclusion, the lingual arteries of both sides are symmetric and run toward inferior and lateral directions from tongue tip to $75 \%$ of total length, but there is no significant difference around base of tongue. Finally the lingual artery located within $50 \%$ of transverse length of tongue from midline raphe. Lingual artery is usually sacrificed during resection of lesion involving tongue base. Distal injury of the lingual artery can be salvaged but proximal injury cause disaster in half portion of the mobile tongue. These findings provide surgeons useful information to set safe surgical margin that does not compromise function of the tongue during oral and base of tongue related surgery. The results of present study are notable because this is the first research to evaluate the histologic features of lingual artery on mobile tongue.

\section{CONFLICT OF INTEREST}

No potential conflict of interest relevant to this article was reported.

\section{ACKNOWLEDGMENTS}

The authors would like to thank those anonymous individuals who generously donated their bodies so that this study could be performed.

\section{REFERENCES}

1. Shangkuan H, XinghaiW, Zengxing W, Shizhen Z, Shiying J, Yishi C. Anatomic bases of tongue flaps. Surg Radiol Anat. 1998;20(2):83-8.

2. Hou T, Shao J, Fang S. The definition of the V zone for the safety space of functional surgery of the tongue. Laryngoscope. 2012 Jan;122(1):66-70.

3. Hou TN, Zhou LN, Hu HJ. Computed tomographic angiography study of the relationship between the lingual artery and lingual markers in patients with obstructive sleep apnoea. Clin Radiol. 2011 Jun;66(6):526-9.

4. Kimura Y, Ariji Y, Gotoh M, Toyoda T, Kato M, Kawamata A, et al. Doppler sonography of the deep lingual artery. Acta Radiol. 2001 May;42(3):306-11.

5. Lopez R, Lauwers F, Paoli JR, Boutault F, Guitard J.Vascular territories of the tongue: anatomical study and clinical applications. Surg Radiol Anat. 2007 Apr;29(3):239-44.

6. Lang P, Thrun B, Hofstetter B, Haberl C, Strehlke P, Hagenmaier C, et al. Giant hemangioma of the tongue: combined use of perioperative blood conservation procedures. Anaesthesist. 2002 Jun;51(6): 470-4.

7. Herzog M, Schmidt A, Metz T, Gunthner-Lengsfeld T, Bremert T, Hoppe F, et al. Pseudoaneurysm of the lingual artery after temperature-controlled radiofrequency tongue base reduction: a severe complication. Laryngoscope. 2006 Apr;116(4):665-7.

8. Lins CC, Cavalcanti JS, do Nascimento DL. Extraoral ligature of lingual artery: anatomic and topographic study. Int J Morphol. 2005; 23(3):271-4.

9. Houseman ND, Taylor GI, Pan WR. The angiosomes of the head and neck: anatomic study and clinical applications. Plast Reconstr Surg. 2000 Jun;105(7):2287-313.

10. Johnson RE, Sigman JD, Funk GF, Robinson RA, Hoffman HT. Quantification of surgical margin shrinkage in the oral cavity. Head Neck. 1997 Jul;19(4):281-6. 